\title{
EM BUSCA DA DEFINIÇÃO DE PAUTAS ATUAIS PARA O DELINEAMENTO DE ESTUDOS SOBRE A SAÚDE DA CRIANÇA E DO ADOLESCENTE EM IDADE ESCOLAR
}

\author{
Maria Inês Ferreira de Miranda* \\ Marta Angélica Iossi** \\ Maria das Graças Carvalho Ferriani*** \\ Maria Aparecida Tedeschi Cano****
}

MIRANDA, M.I.F.de; IOSSI, M.A.; FERRIANI, M.das G.C.; CANO, M.A.T. Em busca da definição de pautas atuais para o delineamento de estudos sobre a saúde da criança e do adolescente em idade escolar. Rev.latinoam.enfermagem, Ribeirão Preto, v. 8, n. 6, p. 83-90, dezembro 2000.

O presente trabalho é uma reflexão na busca da definição de pautas atuais para o delineamento de estudos sobre a assistência à saúde da criança e do adolescente em idade escolar, baseado na literatura atual e na discussão entre profissionais das áreas de Saúde e Educação. As pautas: Assistência à saúde da criança e adolescente em idade escolar; Educação, Saúde e Sociedade - transformações históricas atuais; Saúde, Educação e Condições de Vida, levam a alguns questionamentos. Como promover a saúde da criança e do adolescente em idade escolar dentro dos princípios do Sistema Único de Saúde (SUS) e como trabalhar de modo intersetorial, multiprofissional e interdisciplinar as questões de Saúde e Educação, considerando os NPCN? Ao concluir, são propostas ações desde a desmedicalização do fracasso escolar até a mudança de visão pautadas em conceitos e posturas atuais, sem deixar de considerar as transformações que vêm ocorrendo no setor Educação, com a Nova Lei de Diretrizes e Bases e mais recentemente o lançamento e aplicação dos Novos Parâmetros Curriculares Nacionais.

UNITERMOS: saúde, educação

\section{INTRODUÇÃO}

Os Parâmetros Curriculares Nacionais (PCNs) constituem um referencial de qualidade para a educação em todo País. Sua função é orientar e garantir a coerência dos investimentos no sistema educacional, socializando discussões, pesquisas e recomendações, subsidiando a participação de técnicos e professores brasileiros. Não configuram, portanto, um modelo curricular homogêneo e impositivo que se sobreporia à competência políticoexecutiva dos Estados e Municípios, às diversidades socioculturais das diferentes regiões do País ou à autonomia de professores. O conjunto das proposições expressas nos $\mathrm{PCNs}$ responde à necessidade de referenciais a partir dos quais o sistema educacional do País se organize, para garantir que, respeitadas as diversidades culturais, regionais, étnicas, religiosas e políticas, que atravessam uma sociedade múltipla, estratificada e complexa, a educação possa atuar decisivamente no processo de construção da cidadania, tendo como meta o ideal de uma crescente igualdade de direitos entre os cidadãos, baseado nos princípios democráticos (BRASIL/MEC/SEF, 1997).

Adotando essa perspectiva, as problemáticas sociais são integradas na proposta educacional dos PCNs como Temas Transversais. Não constituem novas áreas, mas antes um conjunto de temas que aparecem tranversalizados nas áreas definidas, isto é, permeando a concepção, os objetivos, os conteúdos e as orientações didáticas de cada área, no decorrer de toda a escolaridade obrigatória. A transversalidade pressupõe um tratamento integrado das áreas e um compromisso das relações interpessoais e sociais escolares com as questões que estão envolvidas nos temas, a fim de que haja coerência

\footnotetext{
* Professor Assistente do Departamento de Enfermagem da Universidade Federal de Rondônia

** Aluna de Pós-Graduação Nível Mestrado do Departamento de Enfermagem Materno-Infantil e Saúde Pública da Escola de Enfermagem de Ribeirão Preto da Universidade de São Paulo

*** Professor Titular do Departamento de Enfermagem Materno-Infantil e Saúde Pública da Escola de Enfermagem de Ribeirão Preto da Universidade de São Paulo. Endereço: Av. Bandeirantes, 3900 - Monte Alegre - 14040-902 - Ribeirão Preto - São Paulo - Brasil

**** Professor Associado do Departamento de Enfermagem Materno-Infantil e Saúde Pública da Escola de Enfermagem de Ribeirão Preto da Universidade de São Paulo
} 
entre os valores experimentados na vivência que a escola propicia aos alunos e o contato intelectual com tais valores. Ética, Saúde, Meio Ambiente, Pluralidade Cultural e Orientação Sexual foram os temas eleitos por envolverem problemáticas sociais atuais e urgentes, consideradas de abrangência nacional e até mesmo de caráter universal.

Em nossa visão, um estudo que propõe uma definição de pautas, deve preocupar-se com a busca de novos paradigmas. Esses novos paradigmas para a assistência à saúde da criança e do adolescente em idade escolar, enquanto grupo social, devem basear-se nas discussões e reflexões que considerem: quais são as concepções de criança, adolescente, vida, escola e prática profissional que fundamentam a percepção dos técnicos e dos próprios usuários que trabalham e utilizam os serviços de atenção à saúde da criança e do adolescente em idade escolar? Como promover a saúde da criança em idade escolar dentro dos princípios do SUS? E, finalmente, como trabalhar de modo intersetorial, multiprofissional e interdisciplinar as questões de Saúde e Educação considerando os Novos PCNs como meio para, através da educação, educar para a saúde?

Lembrando a relação de complementaridade entre setores Educação e Saúde e o fato de que qualquer ação direcionada para a assistência à saúde e para a promoção da educação de crianças e adolescentes deverá pautar-se nos PCNs, propomos para a superação dos desafios acima, a definição e discussão das pautas, por nós consideradas atuais: 1) Assistência à saúde da criança e do adolescente em idade escolar - conceitos e histórico; 2) Educação, Saúde e Sociedade - transformações históricas atuais; 3) Saúde, Educação e Condições de Vida.

\section{CRIANÇA E ADOLESCENTES EM IDADE ESCOLAR - CONCEITOS E HISTÓRICO}

A gênese da saúde escolar faz parte de um movimento social amplo, ocorrido na Europa, durante o século XVIII, destacando-se o países: França, Inglaterra e Alemanha, no qual estão inseridas as questões da Saúde e da Educação (FERRIANI, 1991).

Investigando a origem das escolas medievais, constatamos que estas destinavam-se apenas aos clérigos e a uma camada muito particular da sociedade, a aristocracia rural e a uma nobreza.

Nesse período, destacam-se pensadores como Rousseau, considerado um teórico da democracia liberal, que teve grande influência no pensamento educacional da época o qual preconizava uma educação bastante elitista ao indivíduo suficientemente rico para custear um preceptor.
Rousseau defendia a educação de "Homem total e pleno", assim como a educação para a vida, independente da função ou profissão desejada (ARIÈS, 1981).

Sumariando, pode-se afirmar que a educação de classes populares era restrita no século XVIII e, como a infância nas classes populares era um período curto, as crianças eram força de trabalho, precocemente empregadas em fábricas, tecelagens, cumprindo turnos diários de doze a dezesseis horas de trabalho (ARIÈS, 1981).

A leitura que se pode fazer hoje no Brasil, da situação da educação das crianças de classes populares e do trabalho infantil, tão criticado internacionalmente, não difere do exposto acima.

Já no final do século XIX, as relações entre a classe operária e o Estado burguês sofreram transformações. O próprio Estado desenvolveu novas formas de controle ideológico, onde notamos a extensão da escolarização primária e dos serviços de saúde. A Medicina nessa época traz contribuições científicas com os progressos da bacteriologia e a partir daí, adoecer deixava de ser um problema social, passando a ser uma questão de conhecimento. A Puericultura surge na França e para BOLTANSKI (1969) foi a Revolução Pausteriana que permitiu à Puericultura criar uma teoria baseada num saber científico, positivista, eliminando conhecimentos até então dispersos, contraditórios, mal ligados entre si. $\mathrm{O}$ tom dos textos dirigidos para as mães passou de coloquial e amigável a doutoral e imperativo, já que o novo saber médico passava pelo pasteurismo e cientificismo.

A Puericultura a partir de 1884 na França passa a ser ensinada nas escolas públicas para meninas e jovens, objetivando atingir principalmente as "mulheres do povo", pois estas é que necessitavam ser educadas e civilizadas. Além das escolas, a Puericultura tem seu ensino divulgado em instituições filantrópicas e naturalmente nos serviços médicos.

No período histórico da passagem do feudalismo para o mercantilismo, da instalação do modo de produção capitalista e ascensão da burguesia, enquanto classe dominante, surgem três doutrinas médicas, todas caracterizadas pelo viés eminentemente social: a polícia médica, o sanitarismo e a puericultura.

Neste contexto, a tecnização da higiene escolar ganha contribuições científicas e vários trabalhos foram realizados, denunciando problemas de visão, audição ou preocupando-se com a higiene. A institucionalização da higiene no âmbito escolar só ocorreu na primeira década do século XX, tanto na Europa, como em países da América Latina, Japão e Estados Unidos. A evolução da Saúde do Escolar na Alemanha aconteceu de forma rápida onde o Estado concedia com uma mão, enquanto realizava 
o "controle social" com a outra. É nesses projetos de reforma e controle social, que emerge a Saúde Escolar, na forma primeira de inspeção dos ambientes.

No Brasil, no final do século passado, através da denominação Higiene Escolar, introduz-se a Saúde Escolar calcada, sobretudo, no modelo alemão de "Polícia Médica". Esse modelo, visando à reorganização da própria sociedade, propunha organizar os órgãos públicos, fossem de educação ou de saúde, através de ações de higiene escolar, baseadas em princípios higienistas e no pressuposto eugênico - relacionado à preservação e à melhoria da raça - (COLLARES \& MOYSÉS, 1986). Através de um conjunto de normas, utilizava-se o espaço escolar pelo sistema de saúde, com a finalidade de evitar que doenças contagiosas invadissem o referido espaço.

Enfim, por trás da assistência prestada pelos Programas de Higiene Escolar, havia na realidade uma proposta mobilizadora, controladora, de domesticação das classes populares de acordo com os interesses do sistema produtivo.

A concepção higienista encontra-se, até hoje, fortemente arraigada em algumas propostas da área da saúde. É o que se constata, quando se vem tentando o enfrentamento de epidemias - como dengue, cólera, febre amarela, malária e o retorno da tuberculose, através de planos de combate sob forma de campanhas - "dias nacionais" - que tentam focalizar e tratar de maneira isolada tais problemas de saúde.

Assim, ainda hoje, utilizam-se, prioritariamente, os métodos de controle de hábitos de higiene, que remetem ao indivíduo a responsabilidade pelo risco de adoecer. Firma-se do mesmo modo, uma saúde escolar que tem por objetivo ensinar normas de higiene aos alunos e professores como forma básica de não se contraírem doenças, supondo que a prática de bons hábitos, por si só, seria suficiente para o controle dessas epidemias.

A partir dos anos 50, para além das abordagens normativas da higiene escolar, surgem algumas propostas que representam um movimento no sentido da biologização de questões relacionadas à aprendizagem escolar. É nesse contexto que, nas décadas seguintes, tomam vulto as discussões em torno da desnutrição e dos programas de merenda escolar, dos distúrbios neurológicos e das triagens neurológicas, auditivas e visuais, enquanto tarefas da Saúde Escolar (ZANETTA DE LIMA, 1985).

Supunha-se que o baixo rendimento escolar ou a capacidade mental do indivíduo estivessem intimamente, senão exclusivamente, relacionados ao estado nutricional. Afiguram-se os diagnósticos do tipo "distúrbios de aprendizagem" e "disfunção cerebral" que resultaram na proliferação das especialidades na área de Saúde Escolar, passando esses especialistas a serem chamados para fornecerem explicações para o fenômeno do fracasso escolar (COLLARES \& MOYSÉS, 1986).

Na década de 70 a "Medicina Escolar", que antes era denominada Saúde Escolar passa a priorizar os exames físicos feitos de forma massificada, exame clínico obrigatório para o ingresso do aluno na escola, com revisões periódicas durante o ano letivo. Tal procedimento, ao longo dos anos, não se mostrou eficaz: o exame físico feito rotineiramente, por si só, não imprimiu melhor qualidade de saúde e/ou de educação aos escolares. Além disso, essa rotina de exames não se operacionalizou a contento, uma vez que o crescimento urbano gerou aumento do número de escolas que, por sua vez, não foi acompanhado pelo aumento do número de unidades de saúde e de técnicos de saúde que atuavam na medicina escolar (PATTO, 1990).

A insuficiência de serviços de saúde para atender a demanda escolar em crescimento, levou a centrar o atendimento de saúde aos alunos com supostos "déficit de aprendizagem" ou "distúrbios de comportamento". Procuravam-se explicações médicas para essas questões, fortalecendo a visão compartimentalizada e isolada das crianças, assim o processo de medicalização vai tomando corpo e desencadeia-se, concretamente, o atendimento do fracasso escolar.

\section{EDUCAÇÃO, SAÚDE E SOCIEDADE - TRANSFORMAÇÕES HISTÓRICAS ATUAIS}

A eficácia de qualquer trabalho com crianças depende da capacidade em compreendê-las sem nenhuma maquiagem ou paternalismo, em seu contexto de classe e de relações (MORAES, 1996).

O saber e a ciência enfrentam desafios novos, e a assistência à saúde da criança e do adolescente dentro desse saber científico não poderia ficar alheia, necessitando de um novo delineamento em busca de novos paradigmas.

$\mathrm{O}$ exposto acima significa reconhecer que a sociedade e suas instituições estão atravessando uma situação histórica especial que nos obriga, enquanto trabalhadores na área da saúde, a nos empenharmos em profundas reflexões acompanhadas de críticas, reajustes, reposicionamentos e aperfeiçoamento estratégico. Quando se aborda o tema assistência à saúde da criança e do adolescente em idade escolar, deve-se contextualizar a escola nos macro-sistemas Estado e Sociedade.

A intervenção reguladora do Estado cada vez mais minimalista, faz com que passemos a debater a questão em busca de respostas acerca do papel que devemos cumprir, pois o momento vivido traz para dentro da escola, numa relação dialética com a sociedade, senão os seus problemas, as suas conseqüências, tais como a 
violência urbana, a droga, a criança vitimizada, o abuso de todas a formas e a alta morbi-mortalidade por acidentes (114,9 óbitos /100 mil crianças, numa letalidade de 8,2 óbitos por mil acidentes) (UNGLERT et al., 1987). Sem entender essas questões fica quase impossível traçar qualquer diretriz com relação à assistência à saúde da criança e do adolescente em idade escolar.

Entendidas as questões acima, o fracasso escolar (repetência e evasão) deixará de ser explicado de forma singular como a imposta pela medicalização da educação ou a pedagogização da saúde. Por outro lado, um dos discursos que tentam explicar o fracasso escolar é o da conseqüência de baixas condições sócio-econômicas do aluno, que muitas vezes o leva a abandonar a escola e a ingressar no mercado de trabalho, elevando os índices de evasão e repetência.

Em recente pesquisa, MIRANDA et al. (1999) comprovaram que numa amostra de conscritos de Ribeirão Preto (SP), a saída da escola sem o $2^{\circ}$ grau completo é causada por diversos fatores, entre eles a repetência escolar e a falta de estímulo do próprio sistema escolar e da sociedade à qual o aluno pertence. $\mathrm{O}$ discurso hegemônico da necessidade de trabalhar, considerada como maior fator exógeno, foi desmitificada, pois, dos $29,3 \%$ conscritos que não freqüentam mais a escola, só $13,2 \%$ o fizeram para trabalhar.

Mudar, buscando paradigmas reais, a partir de uma visão crítica de nossa própria atuação junto à população (profissionais da saúde e da educação), fará com que possamos assumir um papel de interlocutor entre o saber técnico e o saber popular. Devemos contribuir para a discussão das bases sociais dos setores Educação e Saúde numa em uma conceituação dos mesmos enquanto condições de vida. O estudo das condições de vida e de seus impactos sobre a situação da saúde da população, em geral, e de grupos sociais, em particular, tem merecido atenção crescente no campo da Saúde Coletiva, seja com o intuito de aprimorar teorias e métodos que possam sustentar os estudos de desigualdades e iniqüidades no processo saúde-doença, seja com a preocupação de construir sistemas de monitoramento que permitam a tomada de decisões no âmbito das Políticas de Saúde (BARATA, 1995).

\section{SAÚDE, EDUCAÇÃO E CONDIÇÕES DE VIDA}

As relações entre saúde, educação e condições de vida estão presentes, de forma diversa e com intensidade distinta, nas abordagens mais conceituais e teóricas sobre causalidade em saúde do século XX. Segundo PAIM et al. (1995), o modelo ecológico, formulado por Gordon em 1920, faz analogia do processo saúde/doença com uma balança e incorpora, sob o rótulo de "meio-ambiente social", os aspectos relacionados a salário, moradia, trabalho, renda e acesso a saneamento básico. O mesmo autor diz que o modelo da história natural da doença de Leavell \& Clark que inspirou na década de 50 o preventivismo e o conceito de "campo de saúde" de Lalonde, formulado em 1974 - que introduziu o estilo de vida e os serviços de saúde no modelo de determinação das doenças - ilustram certas tentativas de aproximação do social à saúde da população (PAIM et al., 1995).

Qualquer tentativa de busca de definição de pautas atuais para o delineamento de estudo sobre condições de vida, educação e saúde de um determinado grupo social, passa pela necessária aproximação do social.

\section{Promoção da saúde da criança e do adolescente em idade escolar dentro dos princípios do SUS}

Como promover a saúde da criança em idade escolar dentro dos princípios do SUS? Como trabalhar de modo intersetorial, multiprofissional e interdisciplinar as questões de Saúde e Educação considerando os NPCN? São questionamentos que nos farão buscar respostas baseadas em novos paradigmas e que podem ser vistas em avaliações como as seguintes:

Na avaliação do Programa de Assistência à Saúde do Escolar desenvolvido pela Secretaria Municipal de Saúde da Cidade de Ribeirão Preto/SP (PROASE), realizada por FERRIANI (1994), observa-se como recomendação: a atenção à criança em idade escolar e ao adolescente deve estar contida em uma Politica de Atenção à Saúde da Criança, ou seja, nas ações desenvolvidas pelo sistema de saúde a todas as crianças, pois evasão e o acesso à rede de ensino, ainda são preocupantes em nosso país.

Programas de saúde que atendem somente as crianças matriculadas nas escolas acabam sendo excludentes e não compatíveis com a tão preconizada e falada atenção integral à saúde da criança/adolescente.

No Município de São Paulo, de 1989 a 1992, uma proposta nova de atenção à saúde da criança e do adolescente em idade escolar, significou romper com o modelo assistencialista e clientelista, até então desenvolvido pelo Departamento de Saúde Escolar da Secretaria Municipal de Educação de São Paulo (DSE) e, também, proporcionou o repensar das necessidades de saúde desse grupo etário no interior do conjunto das necessidades de saúde da população. O DSE desenvolvia suas atividades apenas com crianças e adolescentes matriculados na rede municipal de ensino. Ficavam excluídos aqueles da rede estadual e privada e, principalmente as crianças e adolescentes que se 
encontravam fora do sistema educacional, seja porque não tivessem acesso à escola, seja porque já haviam sido expulsas da escola. Para esse contigente de crianças e adolescentes ocorria, assim, uma dupla exclusão: da escola e da atenção à saúde (SUCUPIRA, 1994).

A proposta de atenção à saúde do escolar no contexto do Sistema Único de Saúde emergiu mediante o processo de reorganização dos serviços de saúde que estava baseado nas diretrizes mais gerais da administração municipal - de descentralização e democratização.

Essa proposta baseava-se nos seguintes princípios: 1) previa a participação da população; 2) o ensino de saúde como parte de todas as ações desenvolvidas pela Saúde e pela Educação; 3) as ações coletivas de saúde devem ser de responsabilidade conjunta com a equipe das Unidades Básicas de Saúde e da escola ou creche; 4) a atenção à saúde do escolar é parte integrante da atenção à saúde da criança e do adolescente; a atenção à saúde da criança e do adolescente deve ser integral; 5) Unidade Básica de Saúde é a instância centralizadora e coordenadora das ações coletivas de saúde, constituindo-se na porta de entrada do sistema de saúde para a criança e o adolescente; 6) a participação da família é fundamental na atenção à saúde da criança e do adolescente (SUCUPIRA, 1994).

Ainda no Município de São Paulo, propostas foram definidas na saúde ocular, saúde bucal, saúde auditiva e saúde mental, na vigilância à saúde, nas ações coletivas de saúde, e na atuação de fonoaudiólogos. Nessas propostas foi reforçada a necessidade de se atuar de forma integrada com a escola e outros equipamentos sociais, no sentido de dar retorno sobre a natureza dos problemas encontrados. A proposta implantada, continua na busca de novos paradigmas para a atenção a esse ser em desenvolvimento.

\section{Como trabalhar de modo intersetorial, multiprofissional e interdisciplinar as questões de saúde e educação, considerando os novos PCNs}

$\mathrm{Na}$ incursão pela literatura nos defrontamos com estatísticas que relatam que em 1990, no Brasil, mais da metade da população infanto-juvenil $(58,2 \%)$ era pobre. A situação de pobreza tem efeito direto sobre a vida das crianças e dos adolescentes nos seus aspectos mais fundamentais: saúde, nutrição e educação (FIBGE, 1991). Mediante a complexidade da realidade e considerando que a necessidade da interdisciplinaridade não provém apenas das deficiências do conhecimento científico ou da organização histórica de seu fomento, mas sobretudo da realidade como tal (COHN, 1996), trabalhar de modo intersetorial e multidisciplinar as questões de saúde e educação considerando os Novos Parâmetros Curriculares é o desafio proposto.

Segundo DEMO (1997), para a prática da interdisciplinaridade exigem-se grandes cautelas, entre elas: a) somente pode ser tomada como equipe interdisciplinar aquela composta por especialistas diversificados, de preferência oriundos de áreas "opostas"; b) para funcionar, uma equipe interdisciplinar não pode apenas compor competências diversas, mas sobretudo orquestrar os esforços de modo convergente; não se trata de justapor conhecimentos, mas de integrálos num tecido único; c) a arte de tecer a muitas mãos pode ser auxiliada, no início, pela tática de exigir de cada uma o tecido próprio, para somente depois integrálos; no caso ideal, é mais integrado o que já nasce integrado (...).

Por que as pessoas se reúnem sendo de áreas diferentes? O desafio de inovar na busca de novos paradigmas para a atenção à saúde da criança e do adolescente em idade escolar foi evidenciado em 1997, na proposta contida nos Novos PCNs que fomentam novas discussões, principalmente em relação ao comportamento do professor durante as aulas. Eles vão ter de inserir nas disciplinas gerais, os conceitos de ética, saúde, meio ambiente, orientação sexual e pluralidade cultural - temas transversais - (BRASIL/MEC/SEF, 1997).

Evidencia-se que a formação dos professores deverá ser multidisciplinar, o que ainda não é a prática nas instituições de ensino superior brasileiras.

$\mathrm{Na}$ atenção integral ao desenvolvimento da criança, o conjunto de ações referidas abrange diferentes áreas do conhecimento. Isto significa que deve haver planejamento e supervisão de um trabalho coletivo de natureza multiprofissional. As ações coletivas na área da educação e da saúde podem e devem ser feitas por técnicos com formação multiprofissional e interdisciplinar. A visão multiprofissional deve ser construída de tal forma que cada técnico seja capaz de ter uma concepção integral do indivíduo, sabendo identificar quando há realmente necessidade de atuação conjunta com profissional de outra área.

A necessidade de uma ação interdisciplinar nos setores educação e saúde vem da complementaridade destes. Pesquisas têm demonstrado a importância da escolaridade nas condições de saúde e de cidadania (BRASIL/MEC/SEF, 1997). "Como a escola está sempre inserida numa dada sociedade, muito se tem escrito a respeito das relações desta com aquela. A escola sempre e só reproduz, em sua ação, as características da sociedade na qual esteja inserida; sempre melhora a sociedade, pois a transforma positivamente; é um lugar de luta, de vida e pode reproduzi-la ou não, na dependência da ação concreta nela desenvolvida" (SILVA, 1994).

Desde a construção dos primeiros computadores, 
na metade deste século (condições de vida/tecnologia), novas relações entre conhecimento e trabalho começaram a ser delineadas. Um dos seus efeitos é a exigência de um reequacionamento do papel da educação no mundo contemporâneo, que coloca para a escola um horizonte mais amplo e diversificado (multidisciplinar) do que aquele que, até poucas décadas atrás orientava a concepção e construção dos projetos educacionais. Não basta visar à capacitação dos estudantes para futuras habilitações em termos das especializações tradicionais (multiespecialidade), mas antes trata-se de ter em vista a formação dos estudantes em termos de sua capacitação para a aquisição e o desenvolvimento de novas competências, em função de novos saberes (interdisciplinares) que se produzem e demandam um novo tipo de profissional, preparado para poder lidar com novas tecnologias e linguagem, capaz de responder a novos ritmos e processos. É preciso aprender a aprender ( BRASIL/MEC/SEF, 1997).

Vemos hoje, que a escola está deixando a sua função de formação. Ela é palco da "violência"(desde agressividade até assassinatos), da "prostituição infantil", "da presença de menores vitimizados pela própria família", "da venda e consumo de drogas". Da violência externa à escola à violência interna, devem ser realizados e considerados estudos para quem deseja a busca de pautas que subsidiem os trabalhos sobre as questões que envolvem a assistência à saúde da criança e do adolescente em idade escolar, reequacionando o papel da educação e capacitando profissionais neste mister.

O processo de ajustes econômicos por que passa o País, torna o Estado incapaz em várias áreas: como sobreviver aos cortes orçamentários? As áreas sociais (educação, saúde e segurança pública) são as mais afetadas, complicando o quadro da violência e da mobilidade social, pois sem um setor de educação fortalecido e estruturado, como aspirar a uma profissão melhor e fazer parte de um mercado de trabalho globalizado, com qualidade de vida? Isso tudo só faz aumentar o fosso social existente em nossa sociedade marcada pelas desigualdades, pelas diversidades e pelas diferenças.

MINAYO \& SOUZA (1993), discutindo o crescimento da violência fatal no Rio de Janeiro, na década de 80 , correlacionam esse crescimento à ampliação da crise econômica, com a redução do mercado de trabalho e com o envolvimento precoce da população, com o tráfico e o consumo de drogas.

Com o exposto, reiteramos a necessidade da interdisciplinaridade nas ações e nos estudos, que envolvem a assistência à saúde da criança e do adolescente em idade escolar, pois para essas questões que transcendem o espaço escolar, a sua compreensão e resolução só se darão num envolvimento da Sociedade e do Estado, enquanto co-responsáveis na efetivação de mudanças sociais tão profundas, que vão desde a minimização das diferenças sociais acarretadas pelas políticas de ajustes econômicos, que afetam as condições de vida, até a formação de bons professores e de profissionais comprometidos com as mudanças.

Estabelecemos aqui outro desafio para a efetiva assistência à saúde da criança e do adolescente em idade escolar, pois vemos hoje ações do Ministério da Educação voltadas para a implementação de acervo tecnológico para o ensino fundamental e uma política recessiva para o ensino universitário; toda tecnologia existente, computadores, internet, tele-conferências numa sala de aula, sem um bom professor, tornam-se meras sucatas. $A$ escola será melhor se tiver computadores para o uso dos alunos, com a condição de que tenha bons professores, de preferência portadores de conhecimentos sobre o uso dessas novas ferramentas (BARBIERI \& CARVALHO, 1997).

\section{CONCLUINDO E PROPONDO}

Após o delineamento e reflexão das pautas: Como promover a saúde da criança em idade escolar dentro dos princípios do SUS? Como trabalhar de modo intersetorial, multiprofissional e interdisciplinar as questões de Saúde e Educação considerando os Novos PCNs como meio de, através da educação, educar para a saúde, propomos: 1) Ações no sentido da desmedicalização do fracasso escolar, através da capacitação de recursos humanos numa nova perspectiva: a escolaridade não deve ser encarada como um problema de saúde, mas numa perspectiva interdisciplinar e multidisciciplinar, considerando a saúde como condições de vida, sendo essas condições de vida como específicas de cada classe ou fração de classe social; 2) Estabelecer uma nova relação entre as áreas de Educação e Saúde em busca de práticas, principalmente as que reportem à atuação conjunta junto ao aluno, sua família, escola, comunidade e movimentos populares, buscando uma intersecção entre os programas de saúde do escolar existentes com o Programa de Saúde da Criança da Rede Pública de saúde (SUS), principalmente porque está clara a semelhança de seus objetivos e de suas ações, no que tange ao atendimento integral à saúde da criança e do adolescente. Essas ações que buscam promover a intersecção entre os dois setores, Educação e Saúde, devem ser discutidas e formuladas considerandose as necessidades próprias de cada região (regionalidade/ diversidade/diferenças/desigualdades), tendo como referência o atendimento mais globalizante da saúde do indivíduo e da sociedade numa prática mais educativa, ultrapassando os limites dos consultórios médicos. 3) 
Finalmente, todas as propostas, ações, discussões, que visem à assistência à saúde da criança e do adolescente em idade escolar, além de considerar o descrito acima, deverão pautar-se na consciência de que se trabalha com um objeto/sujeito em desenvolvimento e crescimento.

Considerando MIRANDA (1998), os "conceitos" ou "palavras-chaves": capacitação de recursos humanos, ética, saúde, meio ambiente, pluralidade cultural, desmedicalização, intersecção entre os dois setores Educação e Saúde, intersetorialidade, multiprofissionalidade, interdisciplinaridade, condições de vida, família, escola, comunidade, movimentos populares, contexto micro e macrossistema, relação sujeito-objeto, elementos contextualizadores, dimensões dialéticas internas (interno-biológica e individualpsicológica) e externas (cultural-sociológica e externafísica), tecnologia, deverão pautar todos os estudos no campo da assistência à saúde da criança e do adolescente em idade escolar no momento histórico-social vivido. Momento este que se iguala ao ocorrido na França e Europa com o Iluminismo, no caráter de busca de novas idéias, e ao pós-guerra, com a explosão tecnológica.

\section{IN SEARCH OF A DEFINITION OF CURRENT GUIDELINES FOR OUTLINING STUDIES ON CHILDREN'S AND SCHOOL-AGE ADOLESCENTS' HEALTH}

This work is a reflection of the search for a definition of current guidelines for outlining studies on the assistance to the child's and school-age adolescent's health based on current literature and on the discussion among professionals in the fields of Health and Education. The guidelines: Assistance to the child's and school-age adolescent's health; Education, Health and Society - current historical transformation; Health, Education and Life Conditions lead to the following questions: how to promote the child's and school-age adolescent's health within the principles of the Unified Health System (SUS) and how to deal with issues such as Health and Education in an inter-sectorial, multi-professional and interdisciplinary fashion by taking into account the New National Curricular Standards? In the conclusion, actions are proposed to eradicate the idea that students are at fault in relation to school failure as well as to change the vision based on current concepts and standings by considering all the changes that have taken place in the field of education with the new Law of Bases and Directives and the recent introduction and application of the New National Curricular Standards.

KEY WORDS: health, education

\section{EN BUSCA DE LA DEFINICIÓN DE PAUTAS ACTUALES PARA EL DELINEAMIENTO DE ESTÚDIOS SOBRE LA SALUD DEL NIÑO Y DEL ADOLESCENTE EN EDAD ESCOLAR}

El presente trabajo es una reflexión en la búsqueda de la definición de pautas actuales para el delineamento de estudios sobre la asistencia a la salud del niño y del adolescente en edad escolar, basada en la literatura actual y en la discusión entre profesionales de las áreas de Salud y de la Educación. Las pautas: Asistencia a la salud del niño y adolescente en edad escolar; Educación, Salud y Sociedad -transformaciones históricas actuales; Salud, Educación y Condiciones de Vida, llevan a los interrogantes: Como promover la salud del niño y del adolescente en edad escolar dentro de los principios del Sistema Único de Salud (SUS) y como trabajar de modo intersectorial, multiprofesional, e interdisciplinar los aspectos de la Salud y de la Educación considerando los NPCNs? Concluyendo, se proponen acciones que van desde la desmedicalización del fracaso escolar hasta la mudanza de visión con base en conceptos y posturas actuales, sin dejar de considerar las transformaciones que vienen ocurriendo en el sector de la educación con la nueva ley de directrices/bases y más recientemente, el lanzamiento y aplicación de los Nuevos Parámetros Curriculares Nacionales.

TÉRMINOS CLAVES: salud, educación

\section{REFERÊNCIAS BIBLIOGRÁFICAS}

01. ARIÈS, P. História social da criança e da família. Trad. por Dora Flakman. 2. ed. Rio de Janeiro: Zahar, 1981.279p.

02. BARATA, R.A. Epidemiologia e saber científico. In: CONGRESSO BRASILEIRO DE EPIDEMIOLOGIA, 3; CONGRESSO IBERO-AMERICANO DE EPIDEMIOLOGIA, 2 e CONGRESSO LATINO-AMERICANO DE EPIDEMIOLOGIA ABRASCO,1. Salvador-Bahia, 24 a 28 de abril de 1995. Anais. Salvador, 1995. 18p.
03. BARBIERI, M. R.; CARVALHO, C. P. Formação de professores em tempo de informática. Comunicação e Educação/USP, v. 9, p. 34-39, 1997.

04. BOLTANSKI, L. Prime éducation et morale de classe. Paris: La Haye, 1969. 152p.

05. BRASIL.Ministério da Educação e Cultura. Secretaria de Educação Fundamental. Parâmetros Curriculares Nacionais. Introdução aos parâmetros curriculares nacionais. Brasília, 1997. 
06. COHN, N. Cosmos, caos e o mundo que virá. São Paulo: Companhia das Letras, 1996.

07. COLLARES, C.; MOYSÉS, M. Educação ou saúde? Educação X saúde? Educação e saúde in fracasso escolar-uma questão médica? Cadernos CEDES, n. 15, p. 45-49, 1986.

08. DEMO, P. Conhecimento moderno-sobre ética e intervenção do conhecimento. Petrópolis: Vozes, 1997.

09. FERRIANI, M.G. A inserção do enfermeiro na saúde escolar. São Paulo: EDUSP, 1991.

10. FERRIANI, M. G. Percepções dos atores sociais que utilizam o Programa de Assistência primária da saúde escolar (PROASE) no Município de Ribeirão Preto: análise crítica. Ribeirão Preto, 1994. 181p. Tese (Livre Docência) - Escola de Enfermagem Ribeirão Preto, Universidade de São Paulo.

11. FUNDAÇÃO IBGE. Censo demográfico. Rio de Janeiro: Departamento de Estatística e Indicadores Sociais, 1991. 159p. v. 4.

12. MINAYO, M.C.S.; SOUZA, E. R. Violência para todos. Cadernos de Saúde Pública, v. 9, p. 6578, 1993.

13. MIRANDA, M.I.F. Programa de atenção integral à saúde do escolar: leitura de uma prática/Porto Velho/RO. Ribeirão Preto, 1998. 150p. Dissertação (Mestrado) - Escola de Enfermagem de Ribeirão Preto, Universidade de São Paulo.
14. MIRANDA, M.I.F. et al. Transdisciplinaridade o desafio de inovar. Comunicação e Educação, São Paulo, n. 14, p. 12-20, 1999.

15. MORAES, N.A. Educação e saúde num contexto de crise: seu objeto - seus objetivos. Rio de Janeiro: SME, 1996.

16. PAIM, J.S.; SILVA, L.M.V. da; COSTA,M.C.N.; PRATA, P.R; CESAR, A .L.M. Condições de vida e saúde da população da cidade de Salvador. In: PAIM, J.S. et al. Análise da situação de saúde do Município de Salvador, segundo condições de vida. Salvador: OPS/CNPq-DRC/RCD /63/ 5/12 Relatório Final, v. 1, 1995.

17. PATTO, M.H. A produção do fracasso escolar. São Paulo: T.A. Queiroz, 1990.

18. SILVA, J.M. A criança, a educação e a saúde: a educação escolar . In: CONCEIÇÃO, J.A.N. Saúde escolar a criança e a vida e a escola. São Paulo: Sarvier, 1994.

19. SUCUPIRA. A experiência do município de São Paulo na Atenção a Saúde do Escolar no período de 1989 a 1992. In: CONCEIÇÃO, J.A.N. Saúde escolar, a criança, a vida e a escola. São Paulo: Savier, 1994. (série Monografias Médicas).

20. UNGLERT, C.V.de S. et al. Características epidemiológicas dos acidentes na Infância. Revista de Saúde Pública, v. 21, p. 23-45, 1987.

21. ZANETTA DE LIMA, G. Saúde escolar e educação. São Paulo: CEDES/CORTEZ, 1985. 